\title{
High-mobility thin InSb films grown by molecular beam epitaxy
}

\author{
T. Zhang, S. K. Clowes, M. Debnath, A. Bennett, C. Roberts, J. J. Harris, R. A. Stradling, \\ and L. F. Cohen a) \\ Blackett Laboratory, Imperial College London, Prince Consort Road, London, SW7 2BW United Kingdom \\ T. Lyford and P. F. Fewster \\ PANalytical Research Centre, Sussex Innovation Centre, Brighton, BN1 9SB United Kingdom
}

(Received 12 December 2003; accepted 22 March 2004; published online 14 May 2004)

\begin{abstract}
The problem of preparing high-mobility thin InSb films is revisited for magnetoresistive and spintronic sensor applications. We introduce a growth process that significantly improves the electrical properties of thin unintentionally doped InSb layers $(60-300 \mathrm{~nm})$ epitaxially grown on $\operatorname{GaAs}(100)$ substrates by reducing the density of dislocations within the interfacial layer. The epilayer properties are well described by a differential two-layer model. This model confirms that the contribution of the interface can only be donor-like. Moreover, the electrical properties of the InSb layers change continuously away from the interface up to sample thickness of the order of 1 $\mu \mathrm{m}$. (C) 2004 American Institute of Physics. [DOI: 10.1063/1.1748850]
\end{abstract}

The electrical properties of InSb have been of interest for decades due to the narrow band gap, low effective mass, and high mobility, $\mu$. Interest in this material has been rekindled recently by the discovery of extraordinary magnetoresistance (EMR) in a composite metal-semiconductor structure, which has made possible the fabrication of entirely different types of macroscopic magnetic sensors for read head applications. ${ }^{1,2}$ As the EMR effect at low magnetic field, $\mathbf{B}$, is proportional to $(\mu B)^{2}$, it is desirable to have a material that has high RT mobility. A thin surface-active region is preferred in order to maximize the effective information storage medium bit field. High mobility thin InSb is also interesting for spintronic applications because of the high spin-orbit coupling and large electron $g$ factor $(-50.6)$. Recent measurements of the spin lifetime ${ }^{3}$ in $n$-type degenerate epilayers of InSb are encouraging, suggesting a RT spin lifetime of the order of $300 \mathrm{ps}$.

Unfortunately, InSb itself cannot be used as a substrate due to its very large parallel conduction. Semi-insulating GaAs has been widely employed as the substrate for $\mathrm{InSb}$ growth. ${ }^{4-11}$ The main obstacle to acquiring high mobility of the hybrid system comes from the strain at the InSb/GaAs interface due to the large lattice mismatch of $14.6 \%$, which is particularly detrimental to thin films. Kanisawa et al. ${ }^{12}$ obtained high mobility thin InSb layers on GaAs(111)A substrates by a two-step growth process (referred to as the "NTT recipe"). Nevertheless, this technique failed to improve InSb films grown on $\operatorname{GaAs}(100)$. In this letter, we describe the results of a method for InSb growth by molecular beam epitaxy (MBE). It is greatly helpful to the improvement of unintentionally doped InSb layers $(60-100 \mathrm{~nm})$ directly grown on $\mathrm{GaAs}(100)$ substrates by producing smoother surfaces and reducing the dislocation donor density.

Thin InSb films were deposited on semi-insulating GaAs(100) substrates in a VG Semicon V80 MBE chamber. Reflection high-energy electron diffraction (RHEED) was

\footnotetext{
a) Author to whom correspondence should be addressed; electronic mail: 1.cohen@imperial.ac.uk
}

used for in situ calibration of the V/III flux ratio, i.e., $\mathrm{Sb}_{4} / \mathrm{In}$, and monitoring InSb surface morphology. The growth procedure in this work, referred to as the "Imperial recipe," follows the two-step mode of the NTT recipe with some additional treatment. First of all, a GaAs buffer layer was not required, as it shows no apparent improvement of mobility. The growth rate of InSb was kept at $0.66 \mu \mathrm{m}$ per hour. It has been reported that, for low substrate temperature ( $T_{\text {sub }}$ $<400{ }^{\circ} \mathrm{C}$ ), two-dimensional mirror-like $\mathrm{InSb}$ was obtained under a low V-III flux ratio $(1-2.5),{ }^{4,5}$ This is due to the lower volatility and longer surface lifetime of $\mathrm{Sb}$ compared to As under these circumstances. An optimized $\mathrm{Sb}_{4} / \mathrm{In}$ flux ratio of 1.3 was employed throughout the work as it always provides the highest mobility samples.

The present work lies in the optimization of the LT layer thickness and an additional anneal step. The LT growth of undoped $\mathrm{InSb}(20 \mathrm{~nm})$ was performed at the minimum temperature capable of maintaining binary growth, which in our case was found at $300{ }^{\circ} \mathrm{C}^{13}$ The growth of $20 \mathrm{~nm}$ of InSb at the LT stage always started in the Volmer-Weber mode, i.e., three-dimensional. The RHEED patterns remained spotty and streaky patterns started to appear at the end of the LT stage. The sample was then annealed at a specified temperature for fixed time. Streaky patterns of $(1 \times 3)$ reconstruction were observed. The temperature was then adjusted for the HT growth step. In order to determine the optimum temperature for the HT growth step, a series of 300-nm-thick InSb epilayers was studied as a function of growth temperature by the measurement of RT mobility and full width at half maximum of the x-ray rocking curves. ${ }^{13,14}$ As a result, the HT growth of a set of InSb films (60 nm-2 $\mu \mathrm{m})$, was performed at the optimized temperature of $380^{\circ} \mathrm{C}$. At the conclusion of the growth, the $\mathrm{Sb}_{4}$ flux was sustained until the sample temperature fell to $290{ }^{\circ} \mathrm{C}$. The growth temperature was calibrated by monitoring the "system-measured" temperature at which the surface reconstruction during homoepitaxial growth changed between "pseudo $(1 \times 3)$ " and $(2 \times 4)$ when the samples were cooled from the annealing temperature to that of the HT step. The temperature was also periodically 

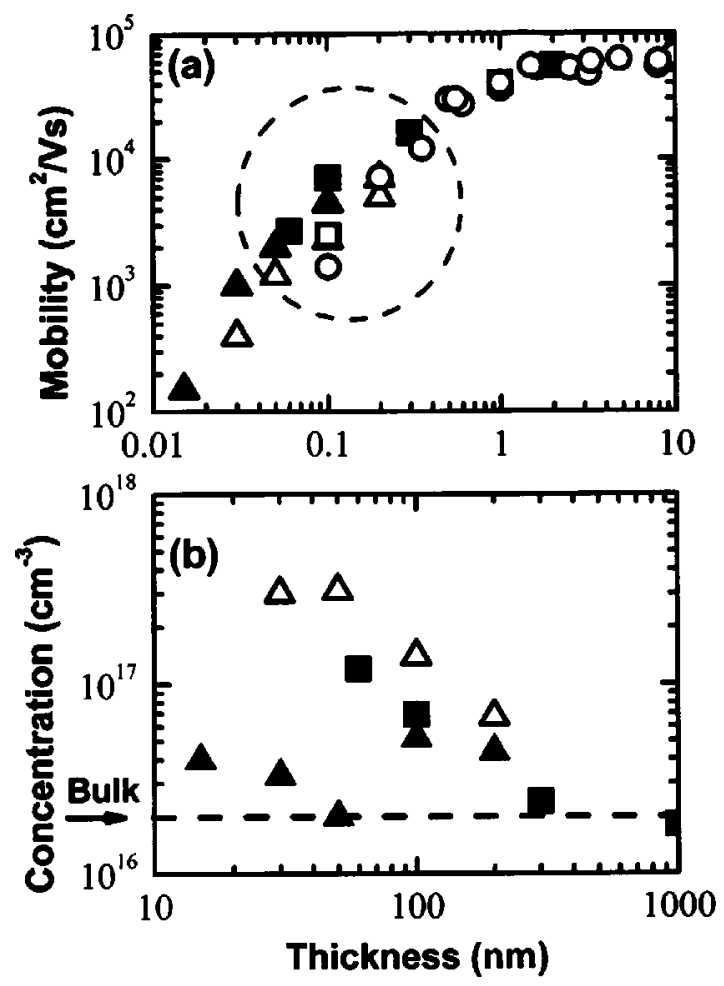

FIG. 1. The apparent RT (a): mobility and (b): carrier concentration of InSb as a function of film thickness using the single-carrier single-layer model. The results of the present work are compared with those of other groups. Symbol keys: solid squares-present work by the Imperial recipe; open square-present work following the NTT recipe; open circles-work presented by other groups (apart from the NTT group); open and solid triangles- InSb grown on $\operatorname{GaAs}(100)$; and $\operatorname{GaAs}(111) \mathrm{A}$, respectively, by the $N T T$ group.

referenced to independent infrared pyrometer measurements. The thickness of the InSb epilayer was calibrated ex situ by glancing incidence x-ray reflectometry, which were in excellent agreement with the target growth thicknesses with differences of $\sim 1.5 \%$. Films were sandblasted to cloverleaf van der Pauw patterns with $5 \mathrm{~mm}$ diameter.

Hall measurements were performed in a magnetic field of $10 \mathrm{mT}$ between RT and $77 \mathrm{~K}$. All samples were $n$ type in the measured temperature range. The relationship between RT mobility and film thickness is summarized in Fig. 1(a), in comparison with those of samples grown elsewhere. ${ }^{4-11}$ All results are calculated using a single-carrier single-layer model. The overall trend shown by the samples is known as the Universal Curve. The samples grown by the Imperial recipe exhibit considerable improvement in the range 60$300 \mathrm{~nm}$. A 100-nm-thick sample (U044) was grown following the NTT recipe and the resulting mobility duplicates the $N T T$ (100) growth result [see Fig. 1(a) —open square symbol], confirming that this method provides a genuine improvement. Atomic force microscope (AFM) images ${ }^{13}$ confirm that the Imperial recipe produces smoother surfaces than the one following the NTT recipe by one order of magnitude with clear atomic growth terraces of height $0.6-0.8$ nm.

The carrier concentration, $n$, calculated from the singlecarrier single-layer model as a function of thickness is shown in Fig. 1(b). Also shown are the NTT results for (111)A and (100) growth, which were extracted using a similar model. The (111)A growth suppresses edge dislocations which are Downloaded 03 Jun 2009 to 131.227.178.130. Redistribution subject to AIP thought to be responsible for the increase of $n$ at the interface. ${ }^{12}$ The lower apparent, i.e., experimentally measured, $n$ from the Imperial (100) growth suggests a lower density of such dislocations, which is consistent with the improved mobility. Note that there is surface depletion because the Fermi level is known to be pinned close to the valence band edge at the surface of the material. Surface depletion will have an increasing role for thinner samples and would increase the apparent $n$ but would not affect the value of the apparent mobility. We do not take the surface depletion into account in the analysis that follows.

A depth profile of the mobility and carrier density as a function of the distance from the interface can be obtained by using a differential approach. ${ }^{14}$ This method assumes that, for a series of $N$ samples of various total thicknesses $t_{n}$, ( $n=1$ to $N$, with all $t_{n}>t_{n-1}$ ), each sample of thickness $t_{n}$ can be considered as consisting of a top layer of thickness $\left(t_{n}-t_{n-1}\right)$, and an underlying layer with the same properties as the sample of thickness $t_{n-1}$. Equations (1) and (2) give the apparent mobility and bulk carrier concentration for the top layer, the subscripts " 1 " and " 2 " denote the contribution of that of the underlying and the top layers, respectively,

$$
\begin{aligned}
& \mu_{2}=\frac{\bar{n} \bar{\mu}^{2} d+S_{q} n_{1} \mu_{1}^{2} d_{1}}{\bar{n} \bar{\mu} d-n_{1} \mu_{1} d_{1}}, \\
& n_{2}=\frac{1}{d_{2}} \frac{\left(\bar{n} \bar{\mu} d-n_{1} \mu_{1} d_{1}\right)^{2}}{\bar{n} \bar{\mu}^{2} d+S_{q} n_{1} \mu_{1}^{2} d_{1}} .
\end{aligned}
$$

Note that $\bar{n}$ and $\bar{\mu}$ are the apparent values extracted from the single-carrier single-layer model, $d$ is the total thickness $\left(d_{1}+d_{2}\right)$, and $S_{q}$ is the sign of the charge of the carriers of the interfacial layer ( +1 for holes and -1 for electrons). The apparent properties of the top layer can then be calculated by taking $\bar{n}$ and $\bar{\mu}$ as the apparent properties of the layer of thickness $t_{n}$, and $n_{1}$ and $\mu_{1}$ as those of the sample of thickness $t_{n-1}$. The depth profile is achieved by repeating this for layers $t_{n-1}$ and $t_{n-2}$, and so on, and the RT results are shown in Fig. 2. It is clear that, above $100 \mathrm{~nm}$ from the interface, the carrier density remains approximately constant at $2 \times 10^{16} \mathrm{~cm}^{-3}$, which is the intrinsic bulk value, but below this thickness the carrier density rapidly increases. This is consistent with the statement that donors arise from dislocation-related states. ${ }^{12}$ There is no corresponding abrupt change in mobility at this thickness, which gradually decreases with decreasing thickness with values $40 \%$ greater than those obtained from single carrier model.

The combination of increasing apparent carrier density and decreasing apparent mobility can be a signature of a $p$-type interfacial layer. ${ }^{15} \mathrm{~A}$ further analysis of the interface is achieved using a two-layer model at high field, which is described as,

$$
\begin{aligned}
\rho_{x y} & =\frac{\sigma_{x y}}{\sigma_{x x}^{2}+\sigma_{x y}^{2}}, \\
\sigma_{x y} & =\frac{n_{s 2} e \mu_{2}^{2} B}{1+\left(\mu_{2} B\right)^{2}}-\frac{S_{q} n_{s 1} e \mu_{1}^{2} B}{1+\left(\mu_{1} B\right)^{2}}
\end{aligned}
$$




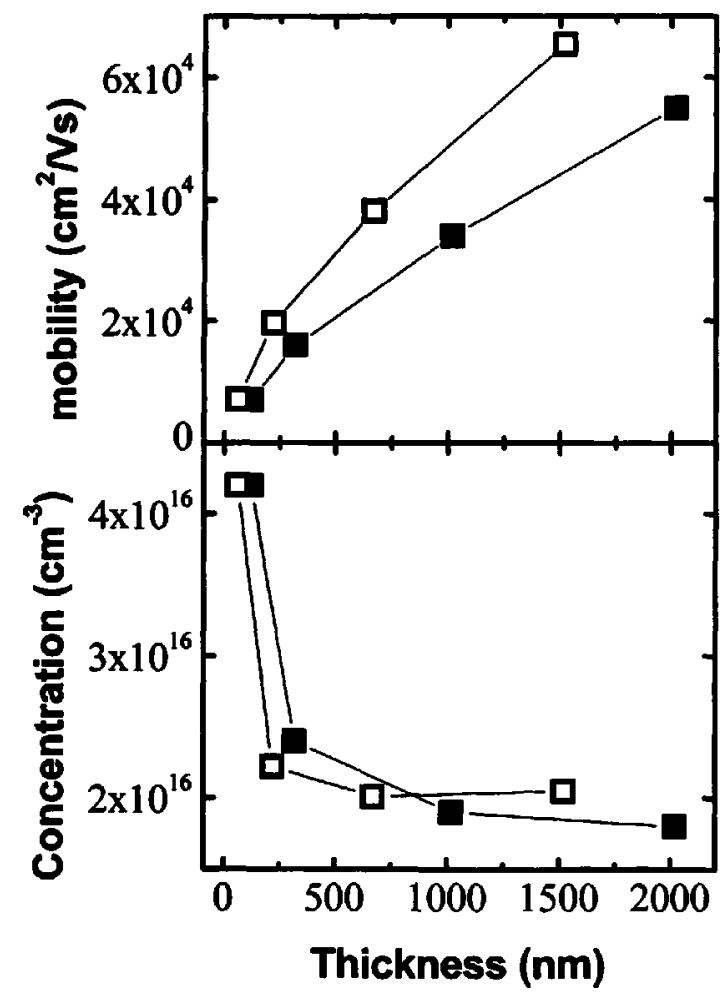

FIG. 2. Fitting of RT mobility and carrier concentration (open squares) as a function of thickness with differential two-layer model. For comparison, the apparent values are-experimental (solid squares) also shown in the same graphs.

$$
\sigma_{x x}=\frac{n_{s 2} e \mu_{2}}{1+\left(\mu_{2} B\right)^{2}}+\frac{n_{s 1} e \mu_{1}}{1+\left(\mu_{1} B\right)^{2}},
$$

where $\rho_{x y}$ is the Hall sheet resistivity, and $\sigma_{x x}$ and $\sigma_{x y}$ are the usual and Hall sheet conductivities, respectively. The subscript 1 now refers to the interfacial layer. Note that sheet concentration, $n_{s}$, are employed in Eqs. (3) and (4), instead of bulk values. Figure 3 shows the experimentally obtained RT values of $\rho_{x y}$ for a $100 \mathrm{~nm}$ InSb film with a $20 \mathrm{~nm} \mathrm{LT}$ interfacial layer. Assuming the interfacial layer is donor-like, a best fit to the experimental data can be easily achieved. This was attained using the constraint that the parameters give a zero field $\rho_{x y}$ equal to that of the experimentally measured value. If the interfacial layer is acceptor-like a best fit to the experiment data could not be achieved, a fit (weighted to the low field region) is shown by the dash line in Fig. 3.

To summarize, the electrical and structural properties of thin InSb films grown on $\mathrm{GaAs}(100)$ by an improved growth method have been investigated. Although three-dimensional island growth cannot be suppressed, we have greatly improved the electrical properties over a thickness range around $100 \mathrm{~nm}$. The two-layer model with its high-field approximation demonstrates that the InSb at the interface with GaAs is donor-like and electrical properties of the layer change con-

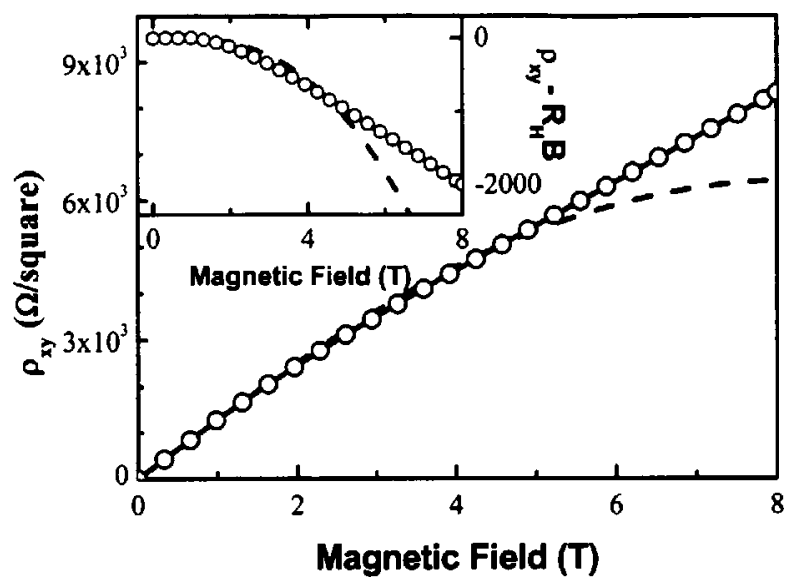

FIG. 3. Experimental RT Hall sheet resistivity (solid circles) for a $100 \mathrm{~nm}$ InSb film with a $20 \mathrm{~nm}$ LT interfacial layer as a function of applied magnetic field, with best fits for donor- (solid line) and acceptor-like (dash line), interfacial layers using a two-layer model at high field. The inset shows $\left(\rho_{x y}-R_{H} B\right)$, where $R_{H}$ is the low field Hall coefficient, as well as its corresponding best fits.

tinuously away from this interface, which is consistent with the expected dislocation density distribution in this material.

The letter is dedicated to Professor R. A. Stradling who passed away after initiating this program. The work is supported by the UK- EPSRC Grant No. GR/R42402 and the EU contract FENIKS: G5RD 200100535.

${ }^{1}$ S. A. Solin, T. Thio, D. R. Hines, and J. J. Heremans, Science 289, 1530 (2000).

${ }^{2}$ S. A. Solin, D. R. Hines, A. C. H. Rowe, J. S. Tsai, Y. A. Pashkin, S. J. Chung, N. Goel, and M. B. Santos, Appl. Phys. Lett. 80, 4012 (2002).

${ }^{3}$ P. Murzyn, C. R. Pidgeon, P. J. Phillips, J. P. Wells, N. T. Gordon, T. Ashley, J. H. Jefferson, T. M. Burke, J. Giess, M. Merrick, B. N. Murdin, and C. D. Maxey, Phys. Rev. B 67, 235202 (2003).

${ }^{4}$ J. I. Chyi, S. Kalem, N. S. Kumar, C. W. Litton, and H. Morkoc, Appl. Phys. Lett. 53, 1092 (1988).

${ }^{5}$ J. E. Oh, P. K. Bhattacharya, Y. C. Chen, and S. Tsukamoto, J. Appl. Phys. 66, 3618 (1989)

${ }^{6}$ S. D. Parker, R. L. Williams, R. Droopad, R. A. Stradling, K. W. J. Barnham, S. N. Holmes, J. Laverty, C. C. Philips, E. Skuras, R. Thomas, X. Zhang, A. Staton-Bevan, and D. W. Pashley, Semicond. Sci. Technol. 4, 663 (1989).

${ }^{7}$ P. E. Thompson, J. L. Davis, J. Waterman, R. J. Wagner, D. Gammon, D. K. Gaskill, and R. Stahlbush, J. Appl. Phys. 69, 7166 (1991).

${ }^{8}$ J. R. Soderstrom, M. M. Cumming, J. Y. Yao, and T. G. Andersson, Semicond. Sci. Technol. 7, 337 (1992)

${ }^{9}$ E. Michel, G. Singh, S. Slivken, C. Besikci, P. Bove, I. Ferguson, and M. Razeghi, Appl. Phys. Lett. 65, 3338 (1994).

${ }^{10}$ X. Weng, R. S. Goldman, D. L. Partin, and J. P. Heremans, J. Appl. Phys. 88, 6276 (2000).

${ }^{11}$ J. Wagner, A. L. Alvarez, J. Schmitz, J. D. Ralston, and P. Koidl, Appl. Phys. Lett. 63, 349 (1993).

${ }^{12}$ K. Kanisawa, H. Yamaguchi, and Y. Kirayama, Appl. Phys. Lett. 76, 589 (2000).

${ }^{13}$ Tong Zhang, M. Debnath, S. K. Clowes, W. Branford, A. Bennett, C. Roberts, L. F. Cohen, and R. A. Stradling, Physica E (Amsterdam) 20, 216 (2004).

${ }^{14}$ C. Mavroidis, J. J. Harris, K. Lee, I. Harrison, B. J. Ansell, Z. Bougrioua, and I. Moerman, Phys. Stat. Sol. 228, 579 (2001), and references therein.

${ }^{15}$ R. A. Stradling, Phys. Scr., T T35, 237 (1991). 